\title{
Object-guided spatial selection in touch without concurrent changes in the
} perceived location of the hands

\author{
Helge Gillmeister ${ }^{1, *}$, Simona Cantarella ${ }^{2}$, Ana Ioana Gheorghiu', and Julia Adler ${ }^{3}$ \\ ${ }^{1}$ Dept. of Psychology, University of Essex \\ ${ }^{2}$ Dept. of Psychology, University of Reading \\ ${ }^{3}$ Department of Psychosomatic Medicine and Psychotherapy, University Medical Center \\ Mainz
}

*Correspondence:

Dept. of Psychology, University of Essex, Wivenhoe Park, Colchester, CO4 3SQ, UK

E-mail: helge@essex.ac.uk

Tel.: +44 (0) 1206873533

Fax.: +44 (0) 1206873801

Submitted to: Experimental Psychology (short research paper) 


\begin{abstract}
In an endogenous cueing paradigm with central visual cues, observers made speeded responses to tactile targets at the hands, which were either close together or far apart, and holding either two separate objects or one common object between them. When the hands were far apart, the response time costs associated with attending to the wrong hand were reduced when attention had to be shifted along one object jointly held by both hands compared to when it was shifted over the same distance but across separate objects. Similar reductions in attentional costs were observed when the hands were placed closer together, suggesting that processing at one hand is less prioritised over that at another when the hands can be 'grouped' by virtue of arising from the same spatial location or from the same object. Probes of perceived hand locations throughout the task showed that holding a common object decreased attentional separability without decreasing the perceived separation between the hands. Our findings suggest that tactile events at the hands may be represented in a spatial framework that flexibly adapts to (object-guided) attentional demands, while their relative coordinates are simultaneously preserved.
\end{abstract}

Keywords: selective attention; endogenous; tactile; spatial; object-guided; body posture; body schema 


\section{Introduction}

Tactile information, such as that received when we grasp a door handle or brush against clothes, or when another person taps us on the shoulder, is initially processed in terms of where on the body it took place as well as its physical dimensions like pressure, vibration, temperature, pain and pleasure. Similar to vision and hearing, information about the identity and the spatial location of tactile stimuli is extracted in parallel, functionally specialised pathways (so-called what and where/how pathways; De Santis, Spierer, Clarke, \& Murray, 2007; Forster \& Eimer, 2004; Reed, Klatzky, \& Halgren, 2005; Van Boven, Ingeholm, Beauchamp, Bikle, \& Ungerleider, 2005). In vision, several studies have demonstrated that these pathways interact with one another in spatial selective attention (e.g. Baylis \& Driver, 1992; Egly, Driver, \& Rafal, 1994; Hollingworth, Maxcey-Richard, \& Vecera, 2012; Marino \& Scholl, 2005; Martinez, Ramanathan, Foxe, Javitt, \& Hillyard, 2007). For example, Egly et al. (1994) presented long outline rectangles either above and below fixation (horizontal objects), or left and right of fixation (vertical objects). To cue covert attentional orienting toward a certain location, the short end of one of the rectangles was briefly highlighted. Egly et al. measured the speed with which participants pressed a single button in response to a subsequent target - the 'filling in' of either the cued end of the rectangle, the uncued end of the same rectangle (same object condition), or the equidistant end of the other, uncued rectangle (different objects condition). It was found that the cost in speed of responding to targets at uncued ends compared to cued ends was greater when the target appeared on another object than when it appeared on the same object, even though their spatial distance from the target was the same. That is, covert attention was shifted more rapidly between spatially separate locations on the same perceptual object than on different objects. In other words, Egly et al. showed that the spread of visual attention is guided by both space- and object-based information. 
Only one study has so far investigated whether a similar organising principle operates in touch. Gillmeister, Adler, and Forster (2010) measured event-related potentials (ERPs) to tactile stimuli at cued and uncued hands when the hands were placed far apart and participants were holding either two separate objects from which the tactile stimuli emanated (far condition), or when these two objects were connected to each other by a bar and thus transformed into one object commonly held by both hands (far object condition). It was found that ERP effects of attention were present earlier in processing when separate objects were held, and later when a common object was held. In fact, attentional modulations in the common object condition were no different from those found when the hands were positioned near one another in space (near condition). That is, tactile processing at one hand was prioritised over that at the other hand at early stages of somatosensory cortical processing (95-150 ms after stimulus onset) when the hands could be treated as separate, unconnected sources of information (far condition). However, no location received prioritised processing at these stages when tactile information from the two hands could be 'grouped' by virtue of arising from the same spatial location or object (near and far object conditions). These findings suggest that, like visual attention, tactile attention applied to one part of an object spreads along object boundaries, strengthening the sensory representation of the entire object. We proposed that such a mechanism may facilitate the bimanual handling of objects.

While Gillmeister et al. (2010) showed that tactile and visual attentional mechanisms operate in similar ways, their study only provided an early cortical index of the modulation of spatial attention by object-based information. It is unknown whether holding common or separate objects affects the speed of attentional orienting or indeed other aspects of body perception. The present study was designed to addresses these questions. Based on Posner's (1978) pioneering examination of endogenous tactile attention, we used central visual cues to direct attention to the left or right hand, and measured the speed of vocal responses to specific 
tactile stimuli (targets) at cued and uncued hands, while other tactile stimuli (nontargets) were to be ignored. Similar to, but extending, Gillmeister at al. (2010), we compared four different conditions: when tactile stimulation arose from two separate objects that observers held in their hands, when the hands were placed either near together (Near) or far apart (Far), and when tactile stimulation arose from a common object held by both hands that were placed either near together (Near Object) or far apart (Far Object). Observers' hands were covered from view, and they were occasionally cued to lift the object(s) they were holding, to reinforce the perception of whether or not tactile event locations were separate or connected. Observers' eye movements were measured to control for any effects of overt orienting toward cued locations.

We hypothesised that, if covert tactile attention cued to a location on an object held by the hand spreads along the boundaries of the object, the cost of attending to an invalidly cued location on an object commonly held by both hands should be smaller than the cost of attending to an equidistant invalidly cued location when the hands are holding two separate objects. This object-guided effect on tactile spatial attention should be greater when hands are positioned far apart in space, compared to when they are positioned near one another, because in the former situation the hands may be treated as spatially separate sources of information while in the latter situation they may be grouped on the basis of their common spatial location.

A further manipulation concerned the nature of the proprioceptive information from the two hands when they are holding one common object versus two separate objects. In Gillmeister et al.'s (2010) far object condition, attentional selection at early stages of cortical processing apparently operated as if the hands were near. This poses the question of whether object-guided tactile attention effects lead to a representation of the hands as 'near' or 'connected', or whether relative distance information is essentially preserved. It has been 
shown repeatedly that tactile-attentional selection as well as tactile temporal order judgments are more effective at greater distances between the hands (e.g. Driver \& Grossenbacher, 1996; Eimer, Forster, Fieger, \& Harbich, 2004; Gillmeister et al., 2010; Shore, Gray, Spry, \& Spence, 2005; Soto-Faraco, Ronald, \& Spence, 2004), even when greater distances are merely illusory by providing false visual feedback (Gallace \& Spence, 2005). While the relative (real or illusory) distance between the hands is known to change the effectiveness of tactile selection, it has never been investigated whether, conversely, the effectiveness of selection changes the perceived distance between tactile event locations. Studies of tactile and body part illusions have found that tactile events and body parts can become mislocalised in the direction of attention (e.g. Austen, Soto-Faraco, Enns, \& Kingstone, 2004; Flach \& Haggard, 2006; Harrar \& Harris, 2009; Kilgard \& Merzenich, 1995), and similarly the perceived distance between the hands may be reduced if they become less attentionally separable through holding a common object. The present study therefore asked participants to indicate on a ruler, the starting point of which was randomly offset for each measurement, the perceived location of their (unseen) left and right index fingers at the start, end, and at regular intervals throughout the attentional task in each condition. If the hypothesised changes in the effectiveness of tactile-attentional selection through holding a common object change the perceived relative locations of tactile events, the reported distance between the hands should be smaller when holding a common object (Far Object) than when holding separate objects (Far). Conversely, the perceived distance should be similar in Far and Far Object conditions if holding the same or separate objects affects spatial attention in touch without changing the current body schema with respect to the functional relationship between the hands. 


\section{Methods}

\section{Participants}

Thirty-four paid participants (17 men, mean age: 25.8 years) were tested in this study. Two participants had to be excluded due to technical difficulties, and a further one was excluded because of excessive eye movements made in the cue-target interval (>80\% of trials). The study was conducted in accordance with the Declaration of Helsinki (1964) and was approved by the local ethics committee. Informed written consent was obtained from each participant prior to testing.

\section{Stimuli and Apparatus}

Participants' hands were placed on a tabletop, holding on to wooden bars with their left and right hands, and with their left and right index fingers placed onto tactile stimulators which were embedded in the bars. In different conditions, the bars, and therefore the hands, were either placed close together but with the bars not touching (Near), close together and with the bars solidly connected to one another (Near Object), far apart (Far), or far apart but solidly connected through an additional bar attached between them (Far Object) (see Figure 1). That is, left and right index fingers were separated by $8 \mathrm{~cm}$ (Near Object), $9 \mathrm{~cm}$ (Near), or $58 \mathrm{~cm}$ (Far and Far Object). The bars were held at a distance of about 30 to $45 \mathrm{~cm}$ from the body. The bars and participants' hands were covered from view by a black board, which was placed about $30 \mathrm{~cm}$ above the tabletop. A small tabletop microphone (Sony ECM-R300) was placed on the cover in front of them to record vocal response latencies. To measure perceived location of the index finger tips, a ruler was temporarily placed on the cover to run from left to right edge (or offset by some amount) at several times throughout the experiment. 
Figure 1 about here

Visual attentional cues were arrows pointing to the left $(<)$ or right $(>)$, indicating the left or right hand as the likely target location. A third type of cue consisted of two vertical lines ( $\|)$ and indicated that participants should briefly lift up the bars. Cues were presented in red ink on a grey background in the centre of a computer screen that was placed behind the cover and in front of the participant. Tactile targets and nontargets were presented using four 12-volt solenoids, driving a metal rod with a blunt conical tip to the fingertips of the left and right index fingers, making contact with the finger whenever a current was passed through the solenoid. Nontargets were 'continuous' stimuli, where the index finger was contacted continuously for $200 \mathrm{~ms}$. Targets were 'flutter' vibrations, consisting of five successive 5-ms pulses separated by 35-ms pauses, and required a vocal response. White noise was played via earphones to mask any sounds made by the tactile stimulators.

\section{Design and Procedure}

The experiment consisted of four blocks, each consisting of 210 trials, corresponding to the four hand distance conditions (Far, Far Object, Near, Near Object), with the order of blocks counterbalanced across participants. Each trial started with the presentation of a black fixation cross on a grey background in the centre of the screen $(500 \mathrm{~ms})$, followed by the directional or lift cue (100 ms). After the inter-stimulus interval (500 ms), a tactile target or nontarget was presented to the left or right index finger $(200 \mathrm{~ms})$, followed by an interval of $1500 \mathrm{~ms}$ during which participants could make a vocal response. Lift cues were never followed by a tactile target 
or nontarget, but by a 2000-ms pause before the onset of the next cue. Otherwise, the inter-trial interval was $1000 \mathrm{~ms}$. The fixation cross remained on the screen at all times except during cue presentation. Participants were instructed to fixate their gaze straight ahead on the fixation cross, to respond vocally ("pa") whenever a target was detected at either the cued or uncued index finger, and to ignore all nontargets.

Directional cues indicated the most likely location for a target to occur. Per block of 210 trials, there were 160 trials in which the directional cue was followed by a target, 40 trials in which the direction cue was followed by a nontarget (catch trials), and ten lift trials. Half the target and nontarget trials indicated the left, the other half the right, index finger as the likely target location. For the 160 target trials, directional cues were valid (indicating the location of the subsequent target) in 120 trials (75\%), and invalid (indicating the other location) in 40 trials (25\%). For the 40 nontarget trials, nontargets were presented with equal probability to cued and uncued locations.

Before the first trial in each block, as well as after trials 53, 105, 158, and 210 (the last trial), participants were prompted to indicate the perceived location of their left and right index finger tips on a ruler. The offset of the ruler was shifted before each judgment (for each finger) by a random amount between 0 and $20 \mathrm{~cm}$ to avoid answers based on memories of previous judgments. Perceived distance was calculated as the difference between the indicated locations of left and right index fingers at each measurement point, taking into account ruler offsets. At the start of the experimental session, participants were given a demonstration of tactile targets and nontargets, a brief test to ensure they could distinguish targets from nontargets, and a 40-trial practice block of the experimental task. Performance feedback was provided at regular intervals throughout each experimental block, after perceived tactile location information was measured. The experimenter ensured that objects were in their assigned locations before the attentional task was resumed. 


\section{EOG Recording}

Horizontal and vertical EOG were recorded bipolarly from the outer canthi of both eyes, and from above and below one of the eyes, respectively, using $\mathrm{Ag}-\mathrm{AgCl}$ electrodes referenced to the forehead. To encourage participants to lift the bars quickly, and to enable monitoring their performance during lift trials, muscle EMG was recorded with additional electrodes placed on the deltoid muscles of the left and right arms. Electrode impedance was kept below $10 \mathrm{k} \Omega$. A Neuroscan Synamps2 system and SCAN 4.5 software (Compumedics, Melbourne, Australia) were used for recording and offline analysis of the EOG data. Amplifier band-pass was $0.01-100 \mathrm{~Hz}$, and digitisation rate was $500 \mathrm{~Hz}$. EOG was filtered off-line with a $30-\mathrm{Hz}$ digital low pass filter ( $24 \mathrm{~dB}$ slope), and epoched and extracted for the 700-ms period from $100 \mathrm{~ms}$ before the onset of the cue to the onset of the tactile (non)target to check for eye movements in this interval. Trials with horizontal eye movements (HEOG exceeding $\pm 40 \mu \mathrm{V}$ relative to the 100 -ms baseline before cue onset) or vertical eye movements, eye blinks or other artefacts (VEOG exceeding $\pm 100 \mu \mathrm{V}$ relative to baseline) were excluded from analysis.

\section{Results}

Participants missed few vocal responses to targets $(<1 \%)$, and erroneously responded to nontargets in $7.7 \%$ of trials. On average, $16.8 \%$ of trials were lost due to horizontal or vertical eye movements. Mean RTs in each attentional and hand distance / object condition were computed from the remaining correct target trials. Mean RTs from the 23 (of 31) participants who showed attentional cueing effects (RTs in valid vs. invalid cueing trials) of $\geq 10 \mathrm{~ms}$ in the condition where such effects were expected to be largest (Far) were subjected 
to repeated-measures ANOVA for the factors cue (valid vs. invalid), distance (hands far vs. near), and object (separate objects held vs. common, connected object held).

\section{Effects of tactile-spatial attention}

Figure 2 below shows effects of attentional cueing across conditions of hand distance and object(s) held. Cueing effects were largest when hands were far apart (Far) and smallest when they were close together (Near). When hands were far but holding a common object (Far Object), cueing effects were very similar to when the hands were close together (Near). Unlike far apart hands, holding a common object between close hands (Near Object) did not reduce the attentional effects found in the Near condition. Instead, effects of cueing here were most similar to those found when holding a common object between hands that were much further apart (Far Object).

Figure 2 here

These observations were confirmed in a repeated-measures ANOVA on the factors cue, distance, and object. There was a main effect of cue $(\mathrm{F}(1,22)=37.9, \mathrm{p}<.001)$ as RTs were faster in valid compared to invalid cueing trials. Importantly, there was an interaction between cue and distance $(\mathrm{F}(1,22)=4.5, \mathrm{p}=.046)$, as well as a three-way interaction between cue, distance, and object $(\mathrm{F}(1,22)=5.9, \mathrm{p}=.024)$. Follow-up ANOVAs for each condition of distance showed that, when hands were far apart, there was an overall main effect of cue $(\mathrm{F}(1,22)=50.3, \mathrm{p}<.001)$, and an interaction between cue and object $(\mathrm{F}(1,22)=4.4, \mathrm{p}=.049)$. Effects of attentional cueing were larger in the Far $(30 \mathrm{~ms})$ than in the Far Object (18 ms) 
condition. When hands were close together, there was a main effect of cue $(F(1,22)=17.5$, $\mathrm{p}<.001)$, but no interaction between cue and object $(\mathrm{F}<1, \mathrm{p}=.380)$, showing that cueing effects did not reliably differ across Near (15 ms) and Near Object (19 ms) conditions.

It is worth noting that mean RTs were overall shorter in the near conditions (377 ms) than in the far conditions (396 ms; the main effect of distance in the overall ANOVA just missed significance, $\mathrm{F}(1,22)=4.0, \mathrm{p}=.057)$, and this may have affected the size of attentional cueing effects found. Such effects may increase with increasing response times, similar to, for example, the effects of spatial compatibility and of automatic imitation (e.g. Brass et al., 2001). Two of our findings argue against this possibility. First, the shortest RTs overall were found in the Near Object condition, but the attention effect in this condition was numerically larger (19 ms) compared to Near (15 ms) and Far Object (18 ms) conditions. Furthermore, an analysis of cueing effects as a function of RT (for each participant, RTs in valid trials were subtracted from those in valid trials, and the result divided by overall mean RT, for each condition of distance and object) showed the same pattern of effects as the analysis of mean RTs described above (an overall interaction between distance and object: $F(1,22)=5.5$, $\mathrm{p}=.028$; as well as a main effect of object in a separate analysis of Far versus Far Object conditions: $\mathrm{F}(1,22)=4.9, \mathrm{p}=.037$, while there was no difference between Near and Near Object conditions: $\mathrm{F}<1, \mathrm{p}=.430)$.

\section{Effects of perceived distance between the hands}

Figure 3 below shows the perceived distance between the left and right index fingertips at different trials in the experimental block for each condition of distance and object. Across all measurement points hands were perceived as closer in the Near Object condition than in the Near condition, as here the connecting of the two objects meant that 
they were somewhat closer together. Importantly, there were no differences across Far and Far Object conditions, that is, hands were not perceived as closer together when they were connected by a common object than when they were holding separate objects at any point of measurement. These observations were confirmed in repeated-measures ANOVAs for each hand distance, for the factors object and time (trials $0,53,105,158,210$ ). When hands were far apart, there were no main effects of object or time, nor an interaction between them (all $\mathrm{F} \leq 1.5, \mathrm{p} \geq .220)$. When hands were close together, a main effect of object $(\mathrm{F}(1,22)=31.3$, $\mathrm{p}<.001)$ showed that hands were perceived as closer together in the Near Object than Near condition, and there was no effect of time or interaction between object and time (all $\mathrm{F} \leq 1.7$, $\mathrm{p} \geq .175)$.

\section{Effects of location judgments and lift trials on attention}

It may be argued that probing perceived hand locations enhances space-based aspects of tactile attention by emphasising the spatial separation between the hands. An additional analysis comparing the 26 trials immediately following location judgments with the 26 trials preceding location judgments, however, showed that effects of cueing, and of hand distance on cueing, did not differ between preceding and following trials (cue $\mathrm{x}$ trial type:

$\mathrm{F}(1,22)=1.6, \mathrm{p}=.227$; cue $\mathrm{x}$ distance $\mathrm{x}$ trial type: $\mathrm{F}<1, \mathrm{p}=.800)$, suggesting that space-based selection was unaffected by drawing attention to relative hand locations. A similar comparison of attentional cueing trials preceding and following lift trials should explore the importance of asking observers to lift the object(s) they are holding on the object-based aspects of tactile attention in a future study designed to test this ${ }^{1}$, especially since aspects of tactile grouping are thought to require the active exploration of stimuli (see Gallace \&

\footnotetext{
${ }^{1}$ An analysis like this was not possible in this, or in our earlier ERP study, since attentional cueing and lift trials were randomly intermixed rather than regularly spaced.
} 
Spence, 2011) and lift trials were introduced deliberately to reinforce haptic object perceptions throughout the task.

\section{General Discussion}

In line with a previous ERP study (Gillmeister et al., 2010), we found that tactile attention is both space- and object-guided. There was a response time cost associated with cueing the wrong hand as the likely target location (space-based attention). This cost was greater when attention had to be shifted over greater distances than when the hands were closer together (further evidence for space-based attention). When hands were far apart, the costs of invalid cueing were greater when attention had to be shifted from one object held by one hand to a separate object held by the other hand, compared to when it was shifted over the same distance but along one commonly held object (object-guided attention). No such effects on attentional costs were found when the hands were closer together, however.

In the first study using spatially informative cues to direct voluntary (endogenous) tactile attention, Posner (1978) reported no space-based attention effects in a task that required the simple, speeded detection of tactile stimuli. However, tactile discrimination tasks similar to the present study, where responses were required upon detection of tactile targets while ignoring tactile nontargets, have found reliable attentional effects (Forster \& Eimer, 2005; Posner, 1978). Posner (1978) suggested that tactile-spatial orienting may be automatic, but appears to operate more slowly than tactile detection, and therefore only affects performance when processing is slowed by the demands of a discrimination task. In line with this, Spence and McGlone (2001) suggested that tactile-spatial attention, both voluntary and reflexive, may be most ideally explored using discrimination tasks, specifically those involving spatial discriminations. The findings from our study, however, indicate that even 
when the task makes no specific spatial demands on tactile processing, a spatially informative cue can automatically orient endogenous attention to potential sites of tactile stimulation. In fact, endogenous spatial attention in touch may be modified more by stimulus set composition than by the spatial nature of the discrimination task. In tasks requiring nonspatial non-discriminatory responses, attentional effects were largest when nontargets were twice as frequent as targets (144 ms, Forster \& Eimer, 2005), smaller when they are half as frequent ( $60 \mathrm{~ms}$, Posner, 1978), and smallest when they are a quarter as frequent (15-30 ms, this study). By contrast, in tasks requiring discriminatory responses, effects of spatial cueing were relatively more similar in magnitude to those reported here $(15-30 \mathrm{~ms})$ irrespective of whether tactile discrimination was spatial (up vs. down judgments: 44-45 ms, Spence, Pavani, \& Driver, 2000) or non-spatial (continuous versus pulsed judgments: 0-38 ms, Chica, Sanabria, Lupiáñez, \& Spence, 2007; 24-55 ms, Spence et al., 2000).

In line with behavioural (Driver \& Grossenbacher, 1996; Soto-Faraco et al., 2004) and ERP studies (Eimer et al., 2004; Gillmeister et al., 2010), but using an endogenous attention paradigm, we have shown that tactile attentional selection is profoundly affected by posture. Our findings suggest that the processing at one hand over another is prioritised more when there is a greater separation between the hands in external space, presumably because they can be treated as separate sources of information. Similarly, it has been observed that greater distances between response keys can attenuate spatial stimulus-response compatibility effects (Heister, Schroeder-Heister, \& Ehrenstein, 1990) and reduce interference effect in a binary key-press version of the colour-word Stroop task (Lakens, Schneider, Jostmann, \& Schubert, 2011), presumably because keeping different response options spatially separate facilitates their cognitive categorisation. The parallel effects of separating task-relevant event locations in external space across different behavioural paradigms and sensory as well as 
stimulus-response modalities suggests that these effects, rather than being restricted to certain types of attentional tasks, may reflect general, supramodal cognitive mechanisms.

The novel finding of our study is that holding a common object between distant hands also reduces the cost of responding to tactile events at an unattended hand, similar to the effects of decreasing hand distance. Interestingly, our effect of object-guided spatial attention in touch (12 ms) is comparable to that reported in vision $(15 \mathrm{~ms})$ in a study using a very similar paradigm (Egly et al., 1994). Our findings suggest that, when the hands can be meaningfully ,grouped ${ }^{\text {ee }}$ and treated as a single source of information, without occupying the same region of space, the prioritisation of processing at one hand over another is similar to when the hands do occupy the same region of space (for a recent review of other grouping effects in touch see Gallace \& Spence, 2011). Interestingly, recent research has shown that spatial proximity and other grouping factors can determine the binding of visual features into object and event files (Frings \& Rothermund, 2011; Reynolds, Kwan, \& Smilek, 2010; van Dam \& Hommel, 2010). For example, an irrelevant stimulus can become associated with the same response as a relevant stimulus if it is perceived as belonging to the same object, but does not affect responding if it is perceived as belonging to a different object, even when their spatial relationship remains unchanged (Frings \& Rothermund, 2011). This implies that the „,belongingness ${ }^{\text {ee }}$ of perceptual events may determine, not just attentional selection, but cognitive processes more broadly. In addition, we have shown that space-based attentional costs are not reduced further by holding a common object when the hands already occupy the same region of space. This suggests that object-based information does not have effects on attentional selection over and above those exerted by spatial information, and also argues against an interpretation of our findings as reflecting unspecific perceptual effects of holding common versus separate objects. 
A second novel finding is that object-based information can affect tactile-spatial selection without leading to a representation of the hands as 'near' or 'connected'. Previous studies have shown that directing attention in space can shift the perceived location of tactile events (Flach \& Haggard, 2006; Kilgard \& Merzenich, 1995; see also Harrar \& Harris, 2009) and of body parts (Austen et al., 2004) toward the attended point. For example, Austen et al. (2004) found visual-tactile congruency effects indexing the mislocalisation of felt touches to fake hands even when the fake hands were covered. This referral of touch may have been due to simply directing visual attention to the locations of the (unseen) fake hands. Our findings show that, unlike spatial attention, object-guided attention does not change the perceived locations of the hands. This suggests that tactile events may be represented within both external spatial coordinates that are modulated by posture and object-based information, and a separate framework in which relative locational (i.e. proprioceptive) information is preserved. Indeed, it may be advantageous to preserve such information, and therefore the perceived size of the held object, e.g. in order to avoid obstacles, while at the same time ensuring that none of the hands receives complete priority of processing.

\section{Acknowledgments}

This research was supported by a University of Essex Research Promotion Fund to HG.

\section{References}

Austen, E. L., Soto-Faraco, S., Enns, J. T., \& Kingstone, A. (2004). Mislocalizations of touch to a fake hand. Cognitive, Affective \& Behavioral Neuroscience, 4, 170-181. 
Baylis, G. C., \& Driver, J. (1992). Visual parsing and response competition: the effect of grouping factors. Perception \& Psychophysics, 51, 145-162.

Brass, M., Bekkering, H., \& Prinz, W. (2001). Movement observation affects movement execution in a simple response task. Acta Psychologica (Amsterdam), 106, 3-22.

Chica, A. B., Sanabria, D., Lupiáñez, J., \& Spence, C. (2007). Comparing intramodal and crossmodal cuing in the endogenous orienting of spatial attention. Experimental Brain Research, 179, 353-364.

De Santis, L., Spierer, L., Clarke, S., \& Murray, M. M. (2007). Getting in touch: segregated somatosensory what and where pathways in humans revealed by electrical neuroimaging. NeuroImage, 37, 890-903.

Driver, J., \& Grossenbacher, P. (1996). Multimodal constraints on tactile spatial attention. In T. Innui, \& J. McClelland (Eds.), Attention and performance, XVI (pp. 209-235). Cambridge, MA: MIT Press.

Egly, R., Driver, J., \& Rafal, R. D. (1994). Shifting visual attention between objects and locations: evidence from normal and parietal lesion subjects. Journal of Experimental Psychology: General, 123, 161-177.

Eimer, M., Forster, B., Fieger, A., \& Harbich, S. (2004). Effects of hand posture on preparatory control processes and sensory modulations in tactile-spatial attention. Clinical Neurophysiology, 115, 596-608.

Flach, R., \& Haggard, P. (2006). The cutaneous rabbit revisited. Journal of Experimental Psychology: Human Perception and Performance, 32, 717-732. 
Forster, B., \& Eimer, M. (2004). The attentional selection of spatial and non-spatial attributes in touch: ERP evidence for parallel and independent processes. Biological Psychology, 66, 1-20.

Forster, B. \& Eimer, M. (2005). Covert attention in touch: behavioral and ERP evidence for costs and benefits. Psychophysiology, 42, 171-179.

Frings, C. \& Rothermund, K. (2011). To be or not to be... included in an event file: integration and retrieval of distractors in stimulus-response episodes is influenced by perceptual grouping. Journal of Experimental Psychology: Learning, Memory, and Cognition, 37, 1209-1227.

Gallace, A. \& Spence, C. (2005). Visual capture of apparent limb position influences tactile temporal order judgments. Neuroscience Letters, 379, 63-68.

Gallace, A. \& Spence, C. (2011). To what extent do Gestalt grouping principles influence tactile perception? Psychological Bulletin, 137, 538-561.

Gillmeister, H., Adler, J., \& Forster, B. (2010). Object-guided spatial attention in touch: holding the same object with both hands delays attentional selection. Journal of Cognitive Neuroscience, 22, 931-942.

Harrar, V., \& Harris, L. R. (2009). Eye position affects the perceived location of touch. Experimental Brain Research, 198, 403-410.

Heister, G., Schroeder-Heister, P., \& Ehrenstein, W. H. (1990). Spatial Coding and SpatioAnatomical Mapping: Evidence for a Hierarchical Model of Spatial StimulusResponse Compatibility. In R. W. Proctor, \& T. G. Reeve (Eds.), Advances in Psychology, 65 (pp. 117-143). North-Holland: Elsevier. 
Hollingworth, A., Maxcey-Richard, A. M., \& Vecera, S. P. (2012). The spatial distribution of attention within and across objects. Journal of Experimental Psychology: Human Perception and Performance, 38, 135-151.

Kilgard, M. P., \& Merzenich, M. M. (1995). Anticipated stimuli across skin. Nature, 373, 663.

Lakens, D., Schneider, I. K., Jostmann, N. B., \& Schubert, T. W. (2011). Telling things apart: The distance between response keys influences categorization times. Psychological Science, 22, 887-890.

Marino, A. C., \& Scholl, B. J. (2005). The role of closure in defining the "objects" of objectbased attention. Perception Psychophysics, 67, 1140-1149.

Martinez, A., Ramanathan, D. S., Foxe, J. J., Javitt, D. C., \& Hillyard, S. A. (2007). The role of spatial attention in the selection of real and illusory objects. Journal of Neuroscience, 27, 7963-7973.

Posner, M. I. (1978). Chronometric explorations of mind. Hillsdale, NJ: Erlbaum.

Reed, C. L., Klatzky, R. L., \& Halgren, E. (2005). What vs. where in touch: an fMRI study. NeuroImage, 25, 718-726.

Reynolds, M., Kwan, D., \& Smilek, D. (2010). To group or not to group: an ecological consideration of the Stroop effect. Experimental Psychology, 57, 275-291.

Shore, D. I., Gray, K., Spry, E., \& Spence, C. (2005). Spatial modulation of tactile temporalorder judgments. Perception, 34, 1251-1262. 
Soto-Faraco, S., Ronald, A., \& Spence, C. (2004). Tactile selective attention and body posture: assessing the multisensory contributions of vision and proprioception. Perception \& Psychophysics, 66, 1077-1094.

Spence, C., \& McGlone, F. P. (2001). Reflexive spatial orienting of tactile attention. Experimental Brain Research, 141, 324-330.

Spence, C., Pavani, F., \& Driver, J. (2000). Crossmodal links between vision and touch in covert endogenous spatial attention. Journal of Experimental Psychology: Human Perception and Performance, 26, 1298-1319.

Van Boven, R. W., Ingeholm, J. E., Beauchamp, M. S., Bikle, P. C., \& Ungerleider, L. G. (2005). Tactile form and location processing in the human brain. Proceedings of the National Academy of Sciences of the United States of America, 102, 12601-12605.

Van Dam, W. O. \& Hommel, B. (2010). How object-specific are object files? Evidence for integration by location. Journal of Experimental Psychology: Human Perception and Performance, 36, 1184-1192. 


\section{Figure captions:}

Figure 1. Experimental setup showing the four conditions of distance and object: Hands positioned far apart and holding separate objects (Far), hands positioned close together and holding separate objects (Near), hands positioned far apart but holding a common object (Far Object), and hands positioned close together and holding a common object (Near Object). Black circles indicate the location of the tactile stimulators embedded in the object(s) on which index fingertips were placed. Hands and object(s) were covered from view.

Figure 2. Mean RTs of correct tactile target detection in all conditions of cue, distance, and object. Error bars indicate standard error of the mean. Asterisks indicate significant interactions between cue and object.

Figure 3. Mean perceived distance between left and right index fingertips across five measurement points in all conditions of distance and object. Error bars indicate standard error of the mean. 
Figure 1.
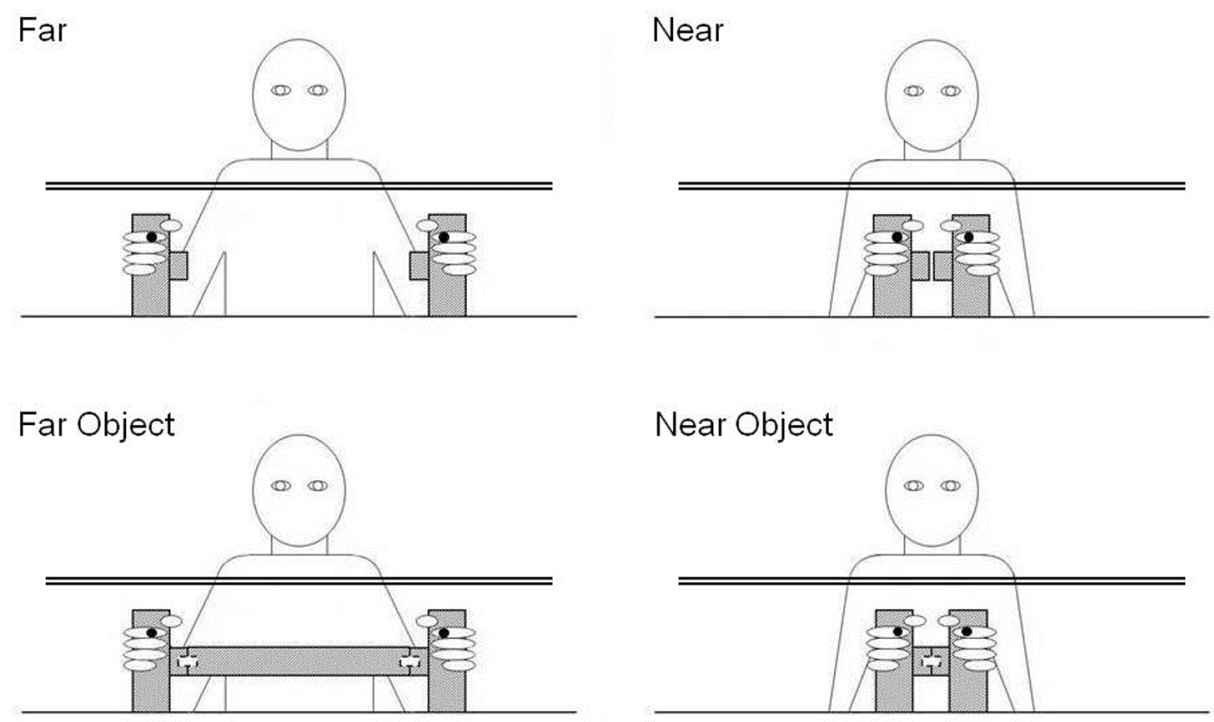

Figure 2.

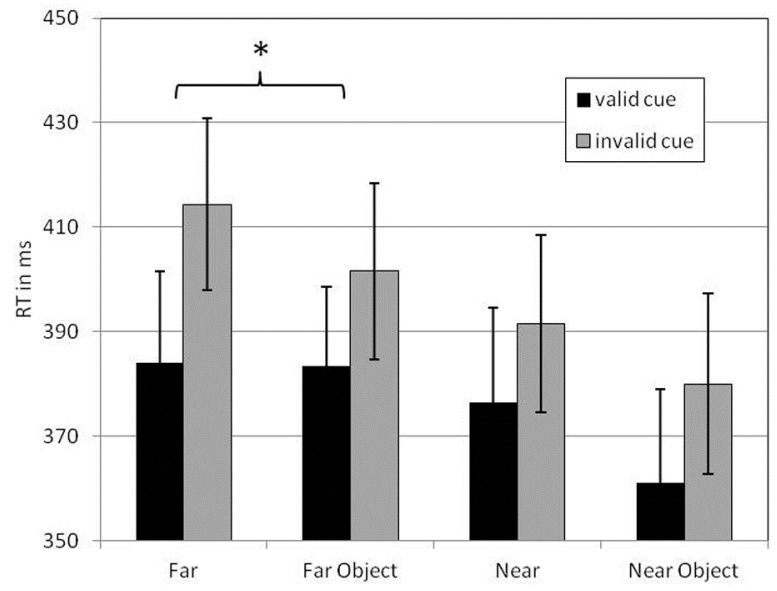

Figure 3. 
Object-guided spatial selection in touch

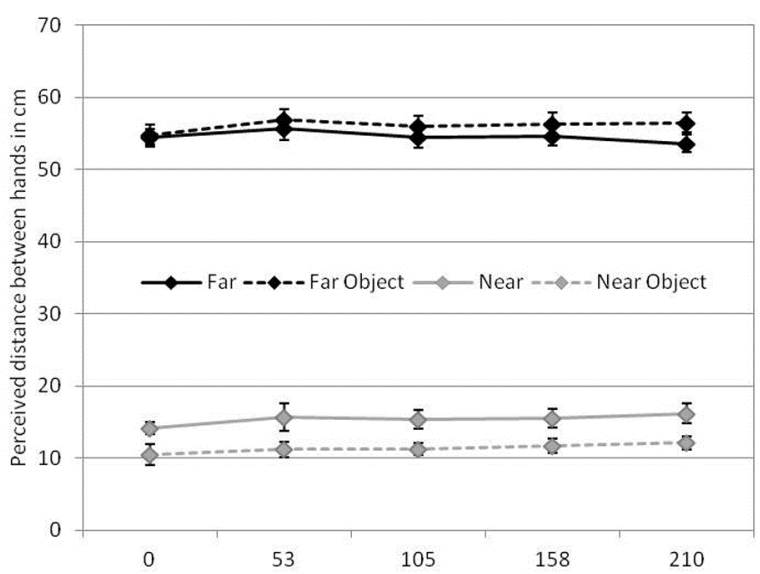

Trial number after which index finger locations were measured 